\title{
A Lattice Algorithm for Pricing Moving Average Barrier Options ${ }^{1}$
}

\author{
Min Dai ${ }^{\mathrm{a}, 2}$, Peifan $\mathrm{Li}^{\mathrm{a}}$, Jin E. Zhang ${ }^{\mathrm{b}}$ \\ ${ }^{a}$ Department of Mathematics, National University of Singapore, Singapore \\ ${ }^{\mathrm{b}}$ School of Economics and Finance, The University of Hong Kong, Pokfulam Road, Hong Kong
}

First Version: January 2008

Final Version: October 2009

Forthcoming in Journal of Economic Dynamics and Control

\begin{abstract}
This paper presents a lattice algorithm for pricing both European- and American-style moving average barrier options (MABOs). We develop a finite-dimensional partial differential equation (PDE) model for discretely monitored MABOs and solve it numerically by using a forward shooting grid method. The modeling PDE for continuously monitored MABOs has infinite dimensions and cannot be solved directly by any existing numerical method. We find their approximate values indirectly by using an extrapolation technique with the prices of discretely monitored MABOs. Numerical experiments show that our algorithm is very efficient.
\end{abstract}

JEL classification: C63; G13

Keywords: Barrier option; Moving average; Lattice algorithm; Forward shooting grid method; Extrapolation

\footnotetext{
${ }^{1}$ We are especially grateful to the three anonymous referees whose helpful comments substantially improved the paper. We also acknowledge helpful comments from Carl Chiarella (editor). Min Dai (MD) is also an affiliated member of Risk Management Institute (RMI), National University of Singapore (NUS). MD has been partially supported by the Singapore MOE AcRF grant (No. R-146-000-096-112) and the NUS RMI grant (No. R-146-000-117/124-720/646). Part of the work was done when MD visited Hong Kong University of Science and Technology and City University of Hong Kong in 2002. Jin E. Zhang (JEZ) thanks Yimin Zhang for special help. JEZ has been partially supported by a grant from the Research Grants Council of the Hong Kong Special Administrative Region, China (Project No. HKU 7549/09H).

${ }^{2}$ Corresponding author. Tel: (65) 6516 2754; fax: (65) 67795452.

E-mail addresses: matdm@nus.edu.sg (M. Dai), lipeifan@nus.edu.sg (P.-F. Li), jinzhang@hku.hk (J.E. Zhang).
} 


\section{Introduction}

Options on the moving average price $^{3}$ (MAP) are widely used in corporate finance. For example, moving average call options are often used to design a poison pill, a strategy in business that increases the likelihood of negative results over positive ones against a party that attempts any kind of takeover. The moving average calls, issued to existing shareholders, would be triggered by the event of a hostile takeover. The French investment bank Compagnie Financiére Indosuez and the French construction company Bouygues have, for example, successfully issued such options/warrants to protect themselves against potentially unfriendly investors, see e.g., Bouaziz, Briys and Crouhy (1994).

Options on the MAP are currently traded as part of structured products in the overthe-counter financial markets. They are particularly welcome by investors who believe in technical analysis ${ }^{4}$. Those investors use the MAP as a measure of market inertia who predict the future trends of a stock with information from its historical MAPs. Therefore, it is very natural to include the MAP as an element in designing financial products to attract such investors. For instance, an investor might make a trading rule that he will buy a stock whenever its 50-day MAP rises above a target level and sell it whenever the MAP drops below this level. A financial institution could issue to the investor an up-and-in call and a down-and-in put triggered by the event that the MAP has hit the target level, which serves as a barrier. Like the normal average in regular Asian options, using the MAP could also effectively alleviate the impact of short-term price fluctuation and protect investors from price manipulation.

\footnotetext{
${ }^{3}$ The moving average price is computed based on the current price and on those in the most recent periods over a fixed length, denoted as $D$ in equation (1) in this paper.

${ }^{4}$ The usefulness of technical analysis has been well justified in the finance literature. For example, Lo, Mamaysky and Wang (2000) find that technical analysis has added value to the investment process based on their novel approach comparing the distribution conditional on technical patterns, such as headand-shoulders and double-bottoms, with the unconditional distribution. Zhu and Zhou (2009) analyze the widely employed moving average trading rule from an asset allocation perspective. They show that technical analysis adds value no matter if stock returns are predictable.
} 
There are a few different ways to use MAP in designing exotic options. The simplest one would be the moving average barrier option (MABO), the up-and-in call and down-and-in put discussed above. The MABO has a regular European option payoff, but is triggered by the event that the MAP hits a prescribed barrier level. The MAP can also be used as an underlying asset in designing options with American, lookback and reset features. A European option on MAP reduces to a known product of a forward starting Asian option or an Asian tail option. In this paper, we focus on the pricing of MABOs and leave the pricing of other MAP-related options for future research.

The study on pricing regular barrier options has a long history. Merton (1973) first studied the price of a down-and-out call option. Conze and Viswanathan (1991) derived the pricing formulas of the up-and-out call and down-and-out put by applying the joint distribution of historical extremum and terminal stock price. Rubinstein and Reiner (1991) provided formulas for many other barrier options. Gao, Huang and Subrahmanyam (2000) proposed an alternative approach for pricing and hedging American barrier options using the decomposition technique. Dai and Kwok (2004) derived analytical formulas for knockin American options. These papers dealt with continuously monitored barrier options. However, in practice, many barrier options traded on markets are discretely monitored. Broadie, Glasserman and Kou (1997) proposed a continuity correction for the discretely monitored barrier option and justified the correction both theoretically and numerically. Kou (2003) extended the results by covering more cases and giving a simpler proof. To resolve the pricing issue of discretely monitored barrier options fully, one has to use a numerical approach. Zvan, Vetzal and Forsyth (2000) presented an implicit method for solving PDE models of contingent claims prices with general algebraic constraints on the solution. Examples of constraints include barriers and early-exercise features. Recently, the literature has evolved into pricing barrier options in a more complicated economy. Fusai and Recchioni (2007) provided an analysis of a quadrature method combined with 
an interpolation procedure for pricing discrete barrier options in GBM, CEV and variance gamma models. Bernard, Le Courtois and Quittard-Pinon (2008) developed a general valuation approach to price barrier options in a stochastic interest-rate environment. Feng and Linetsky (2008) proposed an extrapolation approach for the pricing of barrier options in jump-diffusion models.

Pricing arithmetic Asian options has become a subfield of its own in the discipline of computational finance. The problem is difficult because there is no known closed-form expression for the distribution of the arithmetic average of the lognormal process. For continuously sampled Asian options, Ingersoll (1987) first derived a partial differential equation with $2+1$ dimensions. ${ }^{5}$ Roger and Shi's (1995) transformation uncovered the symmetry of the problem so that they could reduce the problem by one dimension, but it was still nontrivial to solve the resulting $1+1$ dimensional PDE numerically (see Zvan, Forsyth and Vetzal (1998) for flux limiting techniques). Zhang (2001) observed that one difficulty in solving Roger and Shi's PDE came from the singularity embedded in the initial condition. He used a novel approach to remove the singularity in the manner of the perturbation method and obtained highly accurate numerical values of continuous average rate options. The problem indeed has a quasi-analytical solution in a few different forms. Geman and Yor's (1993) analytical expression required calculating inverse Laplace transformations of a confluent hypergeometric function, which was shown by Geman and Eydeland (1995) to be a challenging task. Dufresne's (2000) Laguerre series was mathematically appealing, but it had some problems in the low-volatility case. Linetsky's (2004) elegant spectral expansions of Whittaker functions were alternative representations of Dufresne's Laguerre series. Both Dufresne's and Linetsky's methods were relatively easy to implement in a symbolic system like Maple or Mathematica, but none of them provided a particularly effi-

\footnotetext{
${ }^{5}$ By $2+1$ dimensions, we mean two asset price (stock and cumulative sum) dimensions and one time dimension.
} 
cient way to overcome the low-volatility problem. Because the pricing issue cannot be fully settled by the analytical methods, researchers have tried to develop a few approximation methods. Keman and Vorst's (1990) Monte Carlo simulation with variance reduction and Turnbull and Wakeman's (1991) and Levy's (1992) lognormal approximations were among the earliest attempts at pricing average rate options. Curran's (1994) approximate formula was derived by calculating the expectation conditional on the geometrical mean. Milevsky and Posner's (1998) reciprocal gamma and Posner and Milevsky's (1998) shifted-lognormal and shifted-arcsinh-normal approximations were based on the idea of moment-matching. Built on Roger and Shi's (1995) bounds, Thompson (2002) found some new bounds on the value of fixed-strike and floating-strike Asian options. Ju's (2002) accurate approximate formula obtained via Taylor expansion worked for both discrete and continuous Asian options. Zhang (2003) provided higher-order terms of Zhang's (2001) leading approximation with a perturbation method. For discretely sampled Asian options, Večeř (2001) observed that the Asian option is a special case of the option on a traded account. He obtained a $1+1$ dimensional PDE with piecewise continuous coefficients, which can be regarded as a generalization of Roger and Shi's PDE. Benhamou and Duguet's (2003) small dimension PDE for the homogeneous case was equivalent to Večeř's PDE. Nielsen and Sandmann (2003) developed and compared bounds on the pricing formulas. Recently, Boyle and Potapchik (2008) provided an excellent comprehensive survey of current methods for pricing Asian options and computing their sensitivities to the key input parameters. With extensive numerical exercises, they concluded that Ju's (2002) Taylor expansion and Zhang's (2001) semi-analytical method are two of the most efficient methods to calculate the prices and Greeks of arithmetic Asian options. All of these papers studied options on average prices with a fixed starting date, instead of those on the MAP considered in this paper.

Pricing continuously monitored MABOs is notoriously difficult due to the curse of dimensionality. Only Heritage (2002) studied the pricing of European MABOs. He derived 
an approximate pricing formula for the options by working with the expectations. Unfortunately, we will see later that his formula only applies to a certain range of parameter values. Other related literature includes Kao and Lyuu (2003) and Broadie and Cao (2008). Kao and Lyuu (2003) developed a numerical algorithm based on the binomial tree model to price the moving average lookback option and the moving average reset option, which were listed on the Taipei Stock Exchange in 1999. However, their algorithm is potentially very time consuming because the resulting number of computations grows exponentially with the increasing number of time steps. Broadie and Cao (2008) introduced new variance reduction techniques and computational improvements to the Monte Carlo methods for pricing American-style moving window Asian options.

In this paper, we derive the PDE model and implement the forward-shooting grid method (FSGM) to price discretely monitored MABOs. However, we emphasize that it is not straightforward to apply the FSGM to price continuously monitored MABOs. The reason is that the FSGM essentially relies on a PDE model, but no PDE models are available for continuously monitored MABOs. To overcome the difficulty, we make use of an extrapolation technique together with the prices of discretely monitored MABOs to provide an approximation to that of the continuously monitored counterpart. Numerical results are presented to demonstrate the efficiency of our method. In addition, we show that the approximation formula proposed by Heritage (2002) for European-style MABOs works within a certain range of parameter values but loses power when the window horizon enlarges or the barrier level approaches the spot price. We also find that a simple extension of the least squares Monte Carlo approach proposed by Longstaff and Schwartz (2001) yields lower biased prices for American-style MABOs.

This paper makes three contributions to the literature. First, this paper is one of few studies that consider the pricing of MABOs by bringing together the two strands of literature on pricing barrier and Asian options. For discretely monitored MABOs, it is possible 
to solve the problem numerically because the modeling PDE has finite dimensions. For continuously monitored MABOs, the modeling PDE has infinite dimensions and cannot be solved directly by any existing numerical method. Pricing continuously monitored MABOs, especially American-style MABOs, is an extremely difficult task. Second, we provide a practical way to approximate the solution to this classically difficult computational problem. Our FSGM method for discretely monitored MABOs and our extrapolation method for continuously monitored MABOs are shown to be very efficient. Third, our numerical results can be used by financial institutions and investors as references to determine the premia of options embedded in a moving average trading rule discussed above. Our numerical values can be also used by researchers as a benchmark to test the accuracy of approximate formulas developed based on Heritage (2002) with some other analytical methods. Finding a more accurate analytical approximation for the price of MABOs is an interesting and challenging problem for future research.

The rest of this paper is organized as follows. Section 2 presents PDE formulations for discretely monitored MABOs and explains why PDE models are not available for continuously monitored MABOs. Numerical algorithms are introduced in Section 3. We conduct an extensive numerical study to investigate the performance of our algorithms in Section 4. Section 5 concludes with a short summary.

\section{PDE models for MABOs}

As usual, we assume that the risk-neutral process of the underlying asset price, $S_{t}$, is governed by

$$
\frac{d S_{t}}{S_{t}}=r d t+\sigma d W_{t}
$$

where $r$ and $\sigma$ represent the riskless rate and the volatility, respectively, and $W_{t}$ is a standard one-dimensional Brownian motion. 
Without loss of generality, we consider an up-and-out moving average barrier call option with maturity $T$ and strike price $X$. Let $H=S_{0} e^{b}$ be the predetermined barrier level and $D=T / M$ be the rolling time window for averaging, where $M$ is a positive integer. At time $t$, the arithmetic MAP, $J(t)$, is defined as

$$
J(t)=\frac{1}{D} \int_{t-D}^{t} S_{\tau} d \tau .
$$

Let $\mathcal{T}$ be the set of monitoring time instants at which whether or not the MAP exceeds the barrier level is monitored. If $J(t) \geq H$ for some $t \in \mathcal{T}$, the option is called a "knock-out" and expires worthless; otherwise, the option has the same payoff as a vanilla call option. The terminal payoff of the option can thus be written as

$$
\left(S_{T}-X\right)^{+} \mathbf{1}_{\{J(t)<H, t \in \mathcal{T}\}},
$$

where $\mathbf{1}$ is the indicator function.

We assume regular intervals between adjacent monitoring instants. Let $F$ be the monitoring frequency per window and denote

$$
t_{k}=k D, \quad k=0,1, \cdots, M
$$

We consider the following cases:

i) discrete monitoring with $F=1$ : the monitoring instants are $D, 2 D, \cdots, T$, i.e., $\mathcal{T}=\mathcal{T}_{1}=\left\{t_{k}\right\}_{k=1}^{M}$

ii) discrete monitoring with $F=2$ : the monitoring instants are $D / 2, D, 3 D / 2,2 D, \cdots, T$, i.e., $\mathcal{T}=\mathcal{T}_{2}=\left\{t_{k}-D / 2, t_{k}\right\}_{k=1}^{M}$;

iii) continuous monitoring: $\mathcal{T}=\mathcal{T}_{c}=[0, T]$.

Let us first consider the case of $F=1$.

\subsection{Discrete monitoring with frequency $F=1$}

This case has been considered by Heritage (2002) using a probabilistic approach to obtain an approximate analytical formula. We aim to provide a PDE model and efficient numerical 
methods.

To establish a PDE model, we introduce the following path-dependent variable:

$$
J_{1}(t) \triangleq \frac{1}{t-t_{k-1}} \int_{t_{k-1}}^{t} S_{\tau} d \tau, \quad t \in\left(t_{k-1}, t_{k}\right) \text {, }
$$

for each $k=1,2, \cdots, M$. It is worthwhile pointing out that $J_{1}(t)$ is piecewise continuous. It is continuous within the interval $\left(t_{k-1}, t_{k}\right)$ and discontinuous at $t_{k}$.

By definition, at monitoring time $t_{k}$, the option becomes worthless if $J_{1}\left(t_{k}\right) \geq H$. The option value is thus a function of $S, J_{1}$ and $t$, denoted as $V_{1}\left(S, J_{1}, t\right)$. From (2), we know that

$$
d J_{1}=\frac{S-J_{1}}{t-t_{k-1}} d t
$$

In terms of the Black-Scholes (1973) analysis, it is not difficult to obtain the following governing PDE for European MABOs:

$$
\frac{\partial V_{1}}{\partial t}+\frac{S-J_{1}}{t-t_{k-1}} \frac{\partial V_{1}}{\partial J_{1}}+\frac{1}{2} \sigma^{2} S^{2} \frac{\partial^{2} V_{1}}{\partial S^{2}}+r S \frac{\partial V_{1}}{\partial S}-r V_{1}=0
$$

for $S>0, \quad J_{1}>0, \quad t \in\left(t_{k-1}, t_{k}\right), \quad k=1,2, \cdots, M$. At maturity, we have the terminal condition

$$
V_{1}\left(S, J_{1}, T\right)=(S-X)^{+} \mathbf{1}_{\left\{J_{1}<H\right\}}, \quad \text { for } S>0, J_{1}>0 .
$$

It remains to prescribe a matching condition at $t_{k}$. Apparently,

$$
V_{1}\left(S, J_{1}, t_{k}^{-}\right)=0 \quad \text { if } J_{1} \geq H
$$

On the other hand, when $J_{1}<H$, by continuity we have

$$
V_{1}\left(S_{t_{k}}, J_{1}\left(t_{k}^{-}\right), t_{k}^{-}\right)=V_{1}\left(S_{t_{k}}, J_{1}\left(t_{k}^{+}\right), t_{k}^{+}\right),
$$

which, combined with $J_{1}\left(t_{k}^{+}\right)=\lim _{t \rightarrow t_{k}^{+}} \frac{1}{t-t_{k}} \int_{t_{k}}^{t} S_{\tau} d \tau=S_{t_{k}}$, yields

$$
V_{1}\left(S, J_{1}, t_{k}^{-}\right)=V_{1}\left(S, S, t_{k}^{+}\right) \quad \text { if } J_{1}<H \text {. }
$$


Combination of (6) and (7) gives the matching condition at $t_{k}$ :

$$
V_{1}\left(S, J_{1}, t_{k}^{-}\right)=V_{1}\left(S, S, t_{k}^{+}\right) \mathbf{1}_{\left\{J_{1}<H\right\}}
$$

for $S>0, J_{1}>0, k=1,2, \cdots, M-1$. Equations (4), (5) and (8) form a complete pricing model for European up-and-out call MABOs.

For American style options, due to the early exercise feature, (4) is replaced by a variational inequality

$$
\min \left\{-\frac{\partial V_{1}}{\partial t}-\frac{S-J_{1}}{t-t_{k-1}} \frac{\partial V_{1}}{\partial J_{1}}-\frac{1}{2} \sigma^{2} S^{2} \frac{\partial^{2} V_{1}}{\partial S^{2}}-r S \frac{\partial V_{1}}{\partial S}+r V_{1}, V_{1}-(S-X)^{+}\right\}=0
$$

for $S>0, J_{1}>0, t \in\left(t_{k-1}, t_{k}\right), k=1,2, \cdots, M$, and equations (5)-(8) remain unchanged. Francesco, Pascucci and Polidoro (2008) proved the existence of a strong solution to this type of problem. They also showed that the strong solution is a viscosity solution.

\subsection{Discrete monitoring with frequency $F=2$}

Now we consider the case of $F=2$. In addition to $J_{1}(t)$ given in $(2)$, it is necessary to introduce another path-dependent variable

$$
J_{2}(t)=\frac{1}{t-\left(t_{k-1}-\frac{D}{2}\right)} \int_{t_{k-1}-\frac{D}{2}}^{t} S_{\tau} d \tau, \quad t \in\left(t_{k-1}-\frac{D}{2}, t_{k}-\frac{D}{2}\right),
$$

for each $k=1,2, \cdots, M$. The option knocks out provided that $J_{1}\left(t_{k}\right)>H$ or $J_{2}\left(t_{k}-\frac{D}{2}\right)>$ $H$ for some $k=1,2, \cdots, M$. It is worth pointing out that when $t>T-\frac{D}{2}$, there is only the last monitoring time, $t_{k}=T$, remaining and the option value is nothing but $V_{1}\left(S, J_{1}, t\right)$ as given in the case of $F=1$. As a result, we only need to take into consideration the valuation before $T-\frac{D}{2}$ and denote the option value by $V_{2}=V_{2}\left(S, J_{1}, J_{2}, t\right), t<T-\frac{D}{2}$. Apparently, we have the terminal condition

$$
V_{2}\left(S, J_{1}, J_{2}, T-\frac{D}{2}\right)=V_{1}\left(S, J_{1}, T-\frac{D}{2}\right) \mathbf{1}_{\left\{J_{2}<H\right\}}
$$


Let us derive the governing equation. Consider time interval $\left(t_{k-1}, t_{k}\right)$ at which $d J_{1}$ is given by (3) and

$$
d J_{2}= \begin{cases}\frac{S-J_{2}}{t-\left(t_{k-1}-\frac{D}{2}\right)} d t & \text { for } t \in\left(t_{k-1}, t_{k}-\frac{D}{2}\right) \\ \frac{S-J_{2}}{t-\left(t_{k}-\frac{D}{2}\right)} d t & \text { for } t \in\left(t_{k}-\frac{D}{2}, t_{k}\right)\end{cases}
$$

Again, using the Black-Scholes (1973) analysis, we are able to obtain the governing PDE for $V_{2}\left(S, J_{1}, J_{2}, t\right)$ as follows:

$$
\frac{\partial V_{2}}{\partial t}+\frac{S-J_{1}}{t-t_{k-1}} \frac{\partial V_{2}}{\partial J_{1}}+\frac{S-J_{2}}{t-\left(t_{k-1}-\frac{D}{2}\right)} \frac{\partial V_{2}}{\partial J_{2}}+\frac{1}{2} \sigma^{2} S^{2} \frac{\partial^{2} V_{2}}{\partial S^{2}}+r S \frac{\partial V_{2}}{\partial S}-r V_{2}=0
$$

for $S>0, J_{1}>0, J_{2}>0, t \in\left(t_{k-1}, t_{k}-\frac{D}{2}\right), k=1,2, \ldots, M$; and

$$
\frac{\partial V_{2}}{\partial t}+\frac{S-J_{1}}{t-t_{k-1}} \frac{\partial V_{2}}{\partial J_{1}}+\frac{S-J_{2}}{t-\left(t_{k}-\frac{D}{2}\right)} \frac{\partial V_{2}}{\partial J_{2}}+\frac{1}{2} \sigma^{2} S^{2} \frac{\partial^{2} V_{2}}{\partial S^{2}}+r S \frac{\partial V_{2}}{\partial S}-r V_{2}=0
$$

for $S>0, J_{1}>0, J_{2}>0, t \in\left(t_{k}-\frac{D}{2}, t_{k}\right), k=1,2, \cdots, M-1$.

Similarly, we need matching conditions at $t=t_{1}-\frac{D}{2}, t_{1}, t_{2}-\frac{D}{2}, \cdots, t_{M-1}$. Using the same argument as in the case of $F=1$, we have

$$
\begin{gathered}
V_{2}\left(S, J_{1}, J_{2}, t_{k}^{-}\right)=V_{2}\left(S, S, J_{2}, t_{k}^{+}\right) \mathbf{1}_{\left\{J_{1}<H\right\}}, \\
V_{2}\left(S, J_{1}, J_{2},\left(t_{k}-\frac{D}{2}\right)^{-}\right)=V_{2}\left(S, J_{1}, S,\left(t_{k}-\frac{D}{2}\right)^{+}\right) \mathbf{1}_{\left\{J_{2}<H\right\}},
\end{gathered}
$$

for $k=1,2, \cdots, M-1$.

Equations (9)-(13) form a complete model for discretely monitored European MABOs with $F=2$. As in the case of $F=1$, the extension to the American counterpart is straightforward.

\subsection{Discrete monitoring with frequency $F$ and continuous moni- toring}

Let us look at the general case of discrete monitoring with frequency $F$ where $\mathcal{T}=\mathcal{T}_{F}=$ $\left\{t_{k}-\frac{i-1}{F} D, i=1,2, \cdots, F\right.$, and $\left.k=1,2, \cdots, M\right\}$. In this case, we need to introduce 
$F$ path-dependent variables, $J_{i}, i=1,2, \cdots, F$, where

$$
J_{i}(t)=\frac{1}{t-\left(t_{k-1}-\frac{i-1}{F} D\right)} \int_{t_{k-1}-\frac{i-1}{F} D}^{t} S_{\tau} d \tau, \quad t \in\left(t_{k-1}-\frac{i-1}{F} D, t_{k}-\frac{i-1}{F} D\right),
$$

for each $k=1,2, \cdots, M$. Then, the option value depends on $J_{i}, i=1,2, \cdots, F$, as well as $S$ and $t$. By using a similar argument as above, we can thus formulate the pricing problem as an $F+2$ dimensional PDE problem. The details are omitted.

However, the pricing problem of continuously monitored MABOs cannot be formulated as a finite dimensional PDE, because, at time $t$, the option value depends on all past underlying prices in the rolling time window, i.e., $S_{\tau}, \tau \in[t-D, t)$. In fact, it is easy to see that $\overline{\cup_{F=1}^{\infty} \mathcal{T}_{F}}=[0, T]=\mathcal{T}_{c}$. As a result, a continuously monitored MABO can be regarded as the limit case of discretely monitored MABOs as $F$ tends to infinity. The value function of a continuously monitored MABO has to involve an infinite number of state variables. In the subsequent section, we combine extrapolation techniques with FSGM to price the continuously monitored MABOs.

\section{$3 \quad$ Numerical methods}

Due to the lack of closed-form solutions to the PDE models, we resort to numerical solutions.

\subsection{FSGM for discretely monitored MABOs}

We use the case of $F=1$ to illustrate how the FSGM works. To simplify the notation, the average variable, $J_{1}$, is denoted by $A$. For a given $\Delta t$, we fix

$$
\Delta Z=\sigma \sqrt{\Delta t} \quad \text { and } \quad \Delta Y=\frac{\Delta Z}{K}
$$

Here, $K$ is an integer and $1 / K$ represents a quantization parameter for spacing in the average direction. Denote $t_{n}=n \Delta t, \quad n=0,1, \cdots, N$, and let the discrete values of the 
asset price, $S$, and the average price, $A$, be given by

$$
\begin{aligned}
& S_{j}=S_{0} e^{j \Delta Z} \quad \text { and } A_{k}=S_{0} e^{k \Delta Y}, \\
& \quad j=-n, \cdots, n \text { and } k=n K, n K-1, \cdots,-n K,
\end{aligned}
$$

where $S_{0}$ is the initial underlying price.

Let $V_{j, k}^{n}$ be the price of discretely monitored MABOs with $F=1$ at $\left(t_{n}, S_{j}, A_{k}\right)$. Note that monitoring is conducted only at time $m D, m=1, \cdots, M$. Suppose that $S=S_{j}$ and $A=A_{k}$ at a non-monitoring time, $t_{n}=m D+n^{\prime} \Delta t$, where $1 \leq n^{\prime} \leq \frac{D}{\Delta t}-1$. The correlated evolution of $A$ with $S$ over the next time interval $\left[t_{n}, t_{n}+\Delta t\right]$ can be described by

$$
A_{k^{+}}=\frac{n^{\prime} A_{k}+S_{j+1}}{n^{\prime}+1} \text { for up movement and } A_{k^{-}}=\frac{n^{\prime} A_{k}+S_{j-1}}{n^{\prime}+1} \text { for down movement. }
$$

Thus, the binomial tree method can be expressed as

$$
V_{j, k}^{n}=e^{-r \Delta t}\left[p V^{n+1}\left(S_{j+1}, A_{k^{+}}\right)+(1-p) V^{n+1}\left(S_{j-1}, A_{k^{-}}\right)\right],
$$

where $p=\frac{e^{r \Delta t}-d}{u-d}$ and $u=1 / d=e^{\sigma \sqrt{\Delta t}}$. Usually, the values of $A_{k^{ \pm}}$may not exactly fall on the discrete nodes of the lattice. We therefore utilize the following linear interpolation:

$$
\begin{aligned}
\Pi_{A} V^{n+1}\left(S_{j \pm 1}, A_{k^{ \pm}}\right)= & \frac{A_{k_{\text {floor }}^{ \pm}+1}-A_{k^{ \pm}}}{\Delta Y} V^{n+1}\left(S_{j \pm 1}, A_{k_{\text {floor }}^{ \pm}}\right) \\
& +\frac{A_{k^{ \pm}}-A_{k_{\text {floor }}^{ \pm}}}{\Delta Y} V^{n+1}\left(S_{j \pm 1}, A_{k_{\text {floor }}^{ \pm}+1}\right)
\end{aligned}
$$

to replace $V^{n+1}\left(S_{j \pm 1}, A_{k^{ \pm}}\right)$, where $A_{k_{\text {floor }}^{ \pm}}$is the nearest lattice value less than or equal to $A_{k^{ \pm}}$. Then, (16) is modified to

$$
V_{j, k}^{n}=e^{-r \Delta t}\left[p \Pi_{A} V^{n+1}\left(S_{j+1}, A_{k^{+}}\right)+(1-p) \Pi_{A} V^{n+1}\left(S_{j-1}, A_{k^{-}}\right)\right] .
$$

At maturity and the monitoring time points, we prescribe the same terminal and matching conditions as given in the PDE model. This is the so-called FSGM.

The above algorithm can be readily extended to the case of $F>1$. However, as a lattice algorithm, the FSGM has an amount of computation that explodes as $F$ increases. In the 
case of $F=1$, the memory demand for implementing the FSGM should be $O\left(K N^{2}\right)$, which results from the $(2 N+1)$ grid points in the $S$ direction and the $(2 K N+1)$ grid points in the $A$ direction. The computational cost at the $n$-th time step is $O\left(K n^{2}\right)$, which means that the total computational cost for the FSGM is $O\left(K N^{3}\right)$. Generally, for a discretely monitored MABO with monitoring frequency of $F$, the memory demand is $O(N \times K N \times \cdots \times K N)=$ $O\left(K^{F} N^{F+1}\right)$ and the computational cost for the FSGM is $O\left(K^{F} N^{F+2}\right)$. Both the memory demand and the computational cost increase exponentially with the monitoring frequency, $F$, which leads to difficulty in applying FSGM directly for discretely-monitored MABOs with $F \geq 3$.

As in Jiang and Dai $(2000,2004)$, we are able to show by Taylor expansions that with a given linear interpolation, the FSGM is consistent with the corresponding PDE with the truncation error

$$
O\left(\Delta t+\frac{\Delta Y^{2}}{\Delta t}\right)=O\left(\Delta t+K^{-2}\right)
$$

A proof of the above estimate is placed in the Appendix A. Hence, in order to ensure the first order of consistency, it is necessary to choose $K=O\left(\Delta t^{-\frac{1}{2}}\right)$. It then follows that

$$
\Delta Y=\text { const. } \Delta t
$$

which will be used in all numerical examples presented in Section 4.

We would like to point out that if a quadratic interpolation is adopted in (17), then it suffices to take constant $K$ to achieve the first-order consistency. However, as studied by Forsyth, Vetzal and Zvan (2002), the FSGM with quadratic interpolation is no longer a monotonic scheme and its convergence would be problematic.

\subsection{An extrapolation technique for continuously monitored MA- BOs}

Due to the moving feature of the averaging window, the pricing function of a continuously monitored MABO involves an infinite number of state variables, which makes the FSGM 
infeasible. To obtain an approximate price of a continuously monitored MABO, we will combine extrapolation techniques with FSGM.

Our idea is based on the fact that a continuously monitored MABO can be thought of as the limit of discretely monitored MABOs as the monitoring frequency goes to infinity. Let $V_{F}, F=1,2, \cdots$ be the prices of discretely monitored MABOs with monitoring frequency $F$, and let $V$ be the price of the continuously monitored counterpart. It is obvious that

$$
V=\lim _{F \rightarrow \infty} V_{F},
$$

which implies that we can approximate $V$ by $V_{F}$, for some big enough $F$. Unfortunately, it is extremely time consuming to compute $V_{F}$ for a big $F$ using a lattice method. This motives us to adopt a two-point Richardson (1910) extrapolation to accelerate the convergence:

$$
V_{R E 2}=\frac{4 V_{2}-V_{1}}{3}
$$

where $V_{1}$ and $V_{2}$ are computed from the FSGM. An alternative choice ${ }^{6}$ is the three-point Richardson extrapolation,

$$
V_{R E 3}=\frac{9 V_{3}-8 V_{2}+V_{1}}{2}
$$

Generally, the Richardson extrapolation is designed to approximate the limit of function $G(y)$ as $y \rightarrow 0^{+}$, where $G(y)$ has an asymptotic expansion of the form

$$
G(y) \sim G_{0}+\sum_{k=1}^{s} \alpha_{k} y^{\beta_{k}}+O\left(y^{\beta_{s+1}}\right)
$$

for small $y$, with constants $G_{0}, \alpha_{k}$ and $\beta_{k}\left(\beta_{k} \neq 0\right)$ independent of $y \cdot{ }^{7}$ For example, if

$$
G(y)=G_{0}+\alpha y^{2}+O\left(y^{3}\right) \quad \text { as } y \rightarrow 0,
$$

then the two-point extrapolation (18) can result in an $O\left(y^{3}\right)$ approximation of $G_{0}$. If

$$
G(y)=G_{0}+\alpha_{1} y+\alpha_{2} y^{2}+O\left(y^{3}\right) \quad \text { as } y \rightarrow 0,
$$

\footnotetext{
${ }^{6}$ One can also use Aitken's $\Delta^{2}$-process extrapolation that requires four points, see e.g., Sidi (2003).

${ }^{7}$ For illustration, $\alpha_{k}$ and $\beta_{k}$ are confined to be real numbers.
} 
we can choose the three-point extrapolation (19) to achieve an $O\left(y^{3}\right)$ approximation of $G_{0}$.

The selection of extrapolation method relies on the characteristics of the sequence to be accelerated. Unfortunately, it is intractable to analyze the asymptotic behavior of the $\left\{V_{F}, F=1,2, \cdots\right\}$ sequence as $F$ goes to infinity. We will employ numerical experiments to test the performance of the two-point extrapolation method (18). ${ }^{8}$

\section{$4 \quad$ Numerical Results}

We carry out extensive numerical experiments to investigate the performance of our newly proposed numerical algorithms. As before, up-and-out MABO calls are considered.

\subsection{Numerical results for the discretely monitored MABOs with frequency, $F=1$}

We start with the discretely monitored MABOs with frequency, $F=1$. In this case, the length of the monitoring interval is the same as that of the averaging window.

To examine the convergence of the FSGM, we present in Table 1 the values of Europeanand American-style MABOs computed from our FSGM with varying values of four parameters: the relative barrier level ${ }^{9}, b$, the time grid size, $\Delta t$, the average price grid size, $\Delta Y=\sigma \sqrt{\Delta t} / K$ ( $K$ being an integer $)$, and the window length, $D$. For easy comparisons, other parameters of the MABOs are taken to be the same as those in Heritage (2002): the current stock price, ${ }^{10} S=1.0$, the strike price, $X=0.9$, the interest rate, $r=0.06$, the volatility, $\sigma=0.25$, and the time to maturity, $T=1.0$ year. We use the values computed from Monte Carlo (MC) simulations as benchmarks to measure the errors of the numerical results from our FSGM. To ensure the accuracy of the benchmark, our MC simulations are

\footnotetext{
${ }^{8}$ We have also verified the efficiency of the three-point extrapolation (19) and Aitken's $\Delta^{2}$-process by using European MABO prices obtained from Monte Carlo simulation.

${ }^{9}$ As defined in Section 2, the relative barrier level is the logarithm of the ratio between the barrier level, $H$, and the initial stock price, $S_{0}$, i.e., $b=\ln \frac{H}{S_{0}}$.

${ }^{10}$ For brevity, we omit the subscript 0 in $S_{0}$ in presenting our results in this Section.
} 
conducted with a large number of paths and time steps $\left(10^{6}\right.$ paths, 500,000 plus 500,000 antithetics, and 2,000 time steps in each simulation). The standard errors of the MC simulations are smaller than 0.0001. In Table 1, we observe that, for the European MABOs, the results from our FSGM converge very quickly to the true values computed from MC as we reduce the grid sizes from $\Delta t=1 / 100=0.01$ and $\Delta Y=\sigma \sqrt{\Delta t} / 2=1 / 80=0.0125$ to $\Delta t=1 / 1000=0.0001$ and $\Delta Y=\sigma \sqrt{\Delta t} / 10=\sqrt{10} / 4000=0.000790569$. The error of our FSGM results is smaller than 0.0001 if the grid sizes are taken to be $\Delta t=1 / 1000$ and $\Delta Y=\sigma \sqrt{\Delta t} / 10$. For the American MABOs, the results from our FSGM converge equally well. In this case, it is not easy to obtain an accurate result from the MC. Our FSGM results can then be used as benchmarks to measure the errors of the results from other methods. We also observe that the American MABO price (0.1624) is ten times more expensive than the European one (0.0119) for the relative barrier level of $10 \%(b=0.1)$ and a window length of two weeks ( $D=0.04$ year). The early-exercise premium is very high.

In the rest of the paper, without further specifications, our FSGM results for both the European- and the American-style MABOs are computed with grid sizes of $\Delta t=1 / 1000$ and $\Delta Y=\sigma \sqrt{\Delta t} / 10$. The MC results for the European MABOs are computed with $10^{6}$ paths (500,000 plus 500,000 antithetics) and 2,000 time steps in each simulation.

Heritage (2002) derived an approximate pricing formula, i.e., equation (6), in his paper, for European-style MABOs in terms of a regular barrier option price plus a first-order correction. To investigate the accuracy of our FSGM and Heritage's formula, we present in Table 2 the values of the European-style MABOs with monitoring frequency, $F=1$, computed using MC, our FSGM and Heritage's formula. The highly accurate MC results with standard errors smaller than 0.0001 are used as benchmarks. From the numerical results in Table 2, we observe that the prices computed using our FSGM are almost identical to the MC results for a wide range of all parameters. Our newly proposed lattice algorithm, 
FSGM, is indeed very reliable for the case of $F=1$. Heritage's formula performs very well if the window length is as small as three to four days $(D=0.01$ year), but it loses power if the window length becomes larger, such as two weeks ( $D=0.04$ year) or two to three months $(D=0.2$ year). This is not surprising because Heritage's formula, derived by using a Taylor expansion at $D=0$, works only for small $D$. We further observe that the downward bias of Heritage's formula increases as the spot price, $S$, approaches the barrier level, $H$, i.e., $b=\ln \frac{H}{S} \rightarrow 0$. This is due to the singularity at $S=H$ embedded in the regular barrier option price used in Heritage's formula ${ }^{11}$.

To handle the early-exercise feature in pricing American-style options by simulation, Longstaff and Schwartz (2001) proposed a least-squares Monte Carlo (termed LSM in their paper) approach that uses least squares to estimate the conditional expected payoff to the option holder from continuation. The LSM can be easily applied to value the Americanstyle MABOs. We have implemented the LSM by using 100,000 paths (50,000 plus 50,000 antithetics) and 200 time steps in one path, and choosing 1, S, $S^{2}, A, A^{2}, S A, S^{2} A$ and $S A^{2}$ as a set of basis functions. The accuracy of the LSM is examined in Table 3. The converged numerical values computed by our FSGM, having an accuracy of 0.0001 , are used as benchmarks to measure the errors of the LSM results. From the numerical results in Table 3, we observe that the LSM underprices the American MABOs significantly. The downward bias could be as large as $11 \%$ for the case of a low barrier level $(b=0.10)$ and a short window length $(D=0.04 \text { year })^{12}$.

\footnotetext{
${ }^{11}$ In Heritage's $(2002)$ notation, $\Psi^{\prime}(b) \rightarrow+\infty$ as $b \rightarrow 0$, where $\Psi(b)$, the price of a regular barrier option, is a function of the relative barrier level, $b$.

${ }^{12}$ One reason for the downward bias of the LSM in pricing American-style barrier options is that the LSM is sensitive to the number of time steps, $N$. As an experiment, we applied the LSM to pricing regular American-style calls and American-style up-and-out calls without the moving average feature. We observe that the LSM works well for the regular American-style options, but not for the American-style barrier options. The American-style barrier option prices obtained using the LSM tend to the benchmark values obtained with a standard binomial tree method as $N$ increases, but the convergence rate is very slow. Because the regression in the LSM with a large $N$ leads to an unacceptable amount of computation, we conclude that a simple extension of the LSM does not work well for regular American-style barrier options and American-style MABOs. To enhance the performance of the LSM in pricing American-style MABOs,
} 


\subsection{Numerical results for the continuously monitored MABOs, i.e., $F=\infty$}

Now let us move on to the continuously monitored MABOs, i.e., $F=\infty$.

We now examine the performance of the extrapolation technique combined with our FSGM for continuously monitored European-style MABOs. The amount of computation of our FSGM increases exponentially with the value of monitoring frequency. With the capacity constrains of our computer, we have only managed to compute the values of discretely monitored European-style MABOs with monitoring frequency up to $F=2$. We then compute the values of the continuously monitored European-style MABOs by using Richardson's (1910) two-point extrapolation. The results are presented in Table 4 together with Heritage's (2002) formula (on page 59 in his paper) for continuous MABOs. We observe that Richardson's two-point extrapolation with discrete MABO prices obtained using our FSGM gives very accurate values for the continuous MABOs. Compared with the benchmark values computed using the MC, the relative errors of the results computed using our method are within 3\%. Our proposed method of the extrapolation combined with the FSGM provides a feasible way to price continuously monitored MABOs. We also observe that Heritage's (2002) formula always heavily underprices the MABOs. Its relative error is as large as $-75 \%$ for case II with a $10 \%(b=0.1)$ relative barrier level and a one-month $(D=0.1)$ window length. It is an interesting research topic finding a way to improve Heritage's results and to develop more accurate approximate formulas for both discrete and continuous MABOs .

In Table 5, we present values of the continuously monitored American-style MABOs, i.e., $F=\infty$, from the two-point Richardson extrapolation with the discretely monitored American-style MABO prices computed using our FSGM. Since the error of the extrapolation method for the continuous European-style MABOs has been justified in Table 4 
and the error of our FSGM for the discrete American-style MABOs with $F=1$ has been justified in Table 1, it would be reasonable to believe that the values of the continuous American-style MABOs presented in Table 5 are as accurate as those of the continuous European-style MABOs in Table 4, i.e, the relative errors are within 3\%. To the best of our knowledge, no one has presented the values of the continuous American-style MABOs before in the literature due to the difficulties in numerical computation.

It is of interest to explore the performance of our method when the spot price, $S$, is close to the barrier level, $H$, i.e., when $b=\ln (H / S)$ is small, because the regular barrier option pricing formula has a singularity (the first-order derivative of the option price with respect to $b$ becomes $+\infty$ ) at $b=0$. In Table 6 , we present some numerical results as the spot price approaches the barrier level, $b=\ln (H / S)=0.01$. For the continuous Europeanstyle MABOs, the extrapolation combined with our FSGM gives results that are very close to those obtained using the MC. For the continuous American-style MABOs, the values obtained using the extrapolation combined with our FSGM are the only available results.

\section{Concluding Remarks}

This paper is concerned with the pricing of an exotic barrier option, known as the moving average barrier option (MABO), which is triggered by the moving average of the underlying asset price hitting a prescribed barrier level. When the barrier is discretely monitored, we develop a PDE model that allows us to design an efficient numerical scheme. However, no PDE models are available when the barrier is continuously monitored.

In the case of discrete monitoring, we extend the forward shooting grid method (FSGM) to numerically solve the corresponding PDE model. In the case of continuous monitoring, we make use of extrapolation techniques in combination with the prices of discretely monitored MABOs with $F \leq 2$ computed from the FSGM. Numerical results demonstrate the 
reliability and efficiency of our methods.

Finally, we emphasize that it is straightforward to extend our approach to pricing other structured products associated with the moving average. Since the FSGM is essentially equivalent to a certain explicit finite difference scheme, to further improve accuracy, we may design implicit difference schemes that possess better convergence and stability.

\section{Appendix}

\section{A First-order consistency conditions for the FSGM in the PDE model}

Let us take European-style MABOs as an example. First, we ignore the effect of interpolation, and assume that $U(S, A, t)$ is smooth enough and satisfies

$$
U(S, A, t-\Delta t)=e^{-r \Delta t}\left[p U\left(S u, A^{u}, t\right)+(1-p) U\left(S d, A^{d}, t\right)\right], \quad t \in\left(t_{k-1}, t_{k}\right),
$$

where $A^{u}=\frac{\left(t-\Delta t-t_{k-1}\right) A+S u \Delta t}{t-t_{k-1}}$ and $A^{d}=\frac{\left(t-\Delta t-t_{k-1}\right) A+S d \Delta t}{t-t_{k-1}}$. Using Taylor expansions, it is easy to obtain

$$
\left\{\begin{array}{l}
e^{-r \Delta t}[p(u-1)+(1-p)(d-1)]=r \Delta t+O\left(\Delta t^{2}\right) \\
e^{-r \Delta t}\left[p(u-1)^{2}+(1-p)(d-1)^{2}\right]=\sigma^{2} \Delta t+O\left(\Delta t^{2}\right) \\
e^{-r \Delta t}\left[p(u-1)^{3}+(1-p)(d-1)^{3}\right]=O\left(\Delta t^{2}\right) \\
e^{-r \Delta t}\left[p\left(A^{u}-A\right)+(1-p)\left(A^{d}-A\right)\right]=\frac{S-A}{t-t_{k-1}} \Delta t+O\left(\Delta t^{2}\right) \\
e^{-r \Delta t}\left[p(u-1)\left(A^{u}-A\right)+(1-p)(d-1)\left(A^{d}-A\right)\right]=O\left(\Delta t^{2}\right) .
\end{array}\right.
$$

It follows that

$$
\frac{\partial U}{\partial t}+\frac{S-A}{t-t_{k-1}} \frac{\partial U}{\partial A}+\frac{1}{2} \sigma^{2} S^{2} \frac{\partial^{2} U}{\partial S}+r S \frac{\partial U}{\partial S}-r U=O(\Delta t)
$$


Now, we examine the effect of the interpolation error. Let $V(S, A, t)$ be a smooth function satisfying

$$
V(S, A, t-\Delta t)=e^{-r \Delta t}\left[p \Pi_{A} V\left(S u, A^{u}, t\right)+(1-p) \Pi_{A} V\left(S d, A^{d}, t\right)\right] .
$$

For linear interpolation $\Pi_{A}$, we have,

$$
\Pi_{A} V-V=O\left(\Delta Y^{2}\right)
$$

Combining (20)-(23) gives

$$
\frac{\partial V}{\partial t}+\frac{S-A}{t-t_{k-1}} \frac{\partial V}{\partial A}+\frac{1}{2} \sigma^{2} S^{2} \frac{\partial^{2} V}{\partial S}+r S \frac{\partial V}{\partial S}-r V=O\left(\Delta t+\frac{\Delta Y^{2}}{\Delta t}\right)=O\left(\Delta t+K^{-2}\right)
$$

As a result, to ensure the first-order consistency of FSGM to the PDE, we need to take $K=O\left(\Delta t^{-\frac{1}{2}}\right)$. 


\section{References}

[1] Aitken, A.C., 1926. On Bernoulli's numerical solution of algebraic equations. Proceedings of the Royal Society of Edinburgh 46, 289-305.

[2] Benhamou, E., Duguet A., 2003. Small dimension PDE for discrete Asian options. Journal of Economic Dynamics and Control 27 (11-12), 2095-2114.

[3] Bernard, C., Le Courtois O., Quittard-Pinon F., 2008. Pricing derivatives with barriers in a stochastic interest rate environment. Journal of Economic Dynamics and Control $32(9), 2903-2938$.

[4] Black, F., Scholes, M., 1973. The pricing of options and corporate liabilities. Journal of Political Economy 81, 637-654.

[5] Bouaziz, L., Briys E., Crouhy M., 1994. The pricing of forward-starting Asian options. Journal of Banking and Finance 18, 823-839.

[6] Boyle, P., Potapchik A., 2008. Prices and sensitivities of Asian options: A survey. Insurance: Mathematics and Economics 42, 189-211.

[7] Broadie, M., Cao, M.-H., 2008. Improved lower and upper bound algorithms for pricing American options by simulation. Quantitative Finance 8, 845-861.

[8] Broadie, M., Glasserman P., Kou S., 1997. A continuity correction for discrete barrier options. Mathematical Finance 7 (4), 325-349.

[9] Conze, A., Viswanathan, 1991. Path dependent options: The case of lookback options. Journal of Finance 46 (5), 1893-1907.

[10] Cox, J., Ross S., Rubinstein M., 1979. Option pricing: A simplified approach. Journal of Financial Economics 7, 229-263. 
[11] Curran, M., 1994. Valuing Asian and portfolio options by conditioning on the geometric mean price. Management Science 40, 1705-1711.

[12] Dai, M., Kwok, Y.K., 2004. Knock-in American options. Journal of Futures Markets $24(2), 179-192$.

[13] Dufresne, D., 2000. Laguerre series for Asian and other options. Mathematical Finance $10(4), 407-428$.

[14] Feng, L.-M., Linetsky V., 2008. Pricing options in jump-diffusion models: An extrapolation approach. Operations Research 56 (2), 304-325.

[15] Forsyth, P., Vetzal K., Zvan R., 2002. Convergence of lattice and PDE methods for valuing path dependent options using interpolation. Review of Derivatives Research 5, 273-314.

[16] Francesco, M.D., Pascucci A., Polidoro S., 2008. The obstacle problem for a class of hypoelliptic ultraparabolic equations. Proceedings of the Royal Society A, 464, 155176.

[17] Fusai, G., Recchioni M.C., 2007. Analysis of quadrature methods for pricing discrete barrier options. Journal of Economic Dynamics and Control 31 (3), 826-860.

[18] Gao, B., Huang J.-Z., Subrahmanyam M., 2000. The valuation of American barrier options using the decomposition technique. Journal of Economic Dynamics and Control 24 (11-12), 1783-1827.

[19] Geman, H., Eydeland A. 1995. Domino effect: Invert the Laplace Transformation. Risk 8 (4), 65-67.

[20] Geman, H., Yor M., 1993. Bessel process, Asian options, and perpetuities. Mathematical Finance 3 (4), 349-375. 
[21] Heritage, J.P., 2002. Pricing moving average barrier options. Journal of Computational Finance 5 (4), 51-67.

[22] Ingersoll, J.E., 1987. Theory of financial decision making. Totowa, NJ: Rowman \& Littlefield.

[23] Jiang, L., Dai M., 2000. Convergence analysis of binomial tree method for Americantype path-dependent options. Free Boundary Problems: Theory and Applications, I (China, 1999), GAKUTO International Series: Mathematical Sciences and Applications 13, 153-166. Edited by N. Kenmochi. (see a corrected version at SSRN: http://ssrn.com/abstract $=900407)$

[24] Jiang, L., Dai M., 2004. Convergence of binomial tree method for European/American path-dependent options. SIAM Journal on Numerical Analysis 42 (3), 1094-1109.

[25] Ju, N., 2002. Pricing Asian and basket options via Taylor expansion. Journal of Computational Finance 5 (3), 79-103.

[26] Kao, C.-H., Lyuu Y.-D., 2003. Pricing of moving average-type options with applications. Journal of Futures Markets 23 (5), 415-440.

[27] Kemna, A.G.Z., Vorst, A.C.F., 1990. A pricing method for options based on average asset values. Journal of Banking and Finance, 14, 113-129.

[28] Kou, S.G., 2003. On pricing of discrete barrier options. Statistica Sinica 13, 955-964.

[29] Levy, E., 1992. Pricing European average rate currency options. Journal of International Money and Finance 11, 474-491.

[30] Linetsky, V., 2004. Spectral expansions for Asian (average price) options. Operations Research 52 (6), 856-867. 
[31] Lo, A.W., Mamaysky H., Wang J., 2000. Foundations of Technical Analysis: Computational Algorithms, Statistical Inference, and Empirical Implementation. Journal of Finance 55, 1705-1765.

[32] Longstaff, F.A., Schwartz E.S., 2001. Valuing American options by simulation: A simple least-squares approach. Review of Financial Studies 14 (1), 113-147.

[33] Merton, R.C., 1973. Theory of rational option pricing. Bell Journal of Economics and Management Science 4, 141-183.

[34] Milevsky, M.A., Posner S.E., 1998. Asian options, the sum of lognormals, and the reciprocal gamma distribution. Journal of Financial and Quantitative Analysis 33 (3), 409-422.

[35] Nielsen, J.A., Sandmann, K., 2003. Pricing bounds on Asian options. Journal of Financial and Quantitative Analysis 38 (2), 449-473.

[36] Posner, S.E., Milevsky, M.A., 1998. Valuing exotic options by approximating the SPD with higher moments. Journal of Financial Engineering 7 (2), 109-125.

[37] Richardson, L.F., 1910. The approximate arithmetical solution by finite differences of physical problems involving differential equations, with an application to the stress in a masonry dam. Philosophical Transactions of the Royal Society of London, Series A $210,307-357$.

[38] Rogers, L., Shi Z., 1995. The value of an Asian option. Journal of Applied Probability 32, 1077-1088.

[39] Rubinstein, M., Reiner E., 1991. Breaking down the barriers. Risk 4 (8), 28-35.

[40] Sidi, A., 2003. Practical extrapolation methods: Theory and applications. Cambridge University Press. 
[41] Thompson, G.W.P., 2002. Fast narrow bounds on the value of Asian options. Working paper, Centre for Financial Research, Judge Institute of Management Science, University of Cambridge.

[42] Turnbull, S., Wakeman, L., 1991. A quick algorithm for pricing European average options. Journal of Financial and Quantitative Analysis 26, 377-389.

[43] Večeř, J., 2001. A new PDE approach for pricing arithmetic average Asian options. Journal of Computational Finance 4 (4), 105-113.

[44] Zhang, J.E., 2001. A semi-analytical method for pricing and hedging continuously sampled arithmetic average rate options. Journal of Computational Finance, 5 (1), 59-79.

[45] Zhang, J.E., 2003. Pricing continuously sampled Asian options with perturbation method. Journal of Futures Markets, 23 (6), 535-560.

[46] Zhu, Y.Z., Zhou G.F., 2009. Technical analysis: An asset allocation perspective on the use of moving averages. Journal of Financial Economics 92, 519-544.

[47] Zvan, R., Forsyth P.A., Vetzal, K.R., 1998. Robust numerical method for PDE methods of Asian options. Journal of Computational Finance, 1, 39-78.

[48] Zvan, R., Vetzal, K.R., Forsyth P.A., 2000. PDE methods for pricing barrier options. Journal of Economic Dynamics and Control 24 (11-12), 1563-1590. 
Table 1: Values of the European- and American-style MABOs with monitoring frequency, $F=1$ - convergence of our FSGM for different relative barrier levels, $b=\ln (H / S)$, time grid sizes, $\Delta t$, average price grid sizes, $\Delta Y$, and window lengths, $D$

\begin{tabular}{|c|c|c|c|c|c|c|}
\hline \multirow[b]{2}{*}{$b$} & \multirow[b]{2}{*}{$\Delta t$} & \multirow[b]{2}{*}{$\Delta Y$} & \multicolumn{2}{|c|}{$D=0.2$} & \multicolumn{2}{|c|}{$D=0.04$} \\
\hline & & & European & American & European & American \\
\hline \multirow[t]{5}{*}{0.10} & 0.01 & 0.0125 & 0.0220 & 0.1716 & 0.0105 & 0.1576 \\
\hline & 0.005 & 0.00589256 & 0.0232 & 0.1729 & 0.0113 & 0.1603 \\
\hline & 0.0025 & 0.003125 & 0.0237 & 0.1735 & 0.0116 & 0.1616 \\
\hline & 0.00125 & 0.00147314 & 0.0240 & 0.1738 & 0.0118 & 0.1622 \\
\hline & 0.001 & 0.000790569 & 0.0242 & 0.1739 & 0.0119 & 0.1624 \\
\hline $\mathrm{MC}$ & & & $\begin{array}{c}0.0242 \\
(0.00004)\end{array}$ & & $\begin{array}{c}0.0119 \\
(0.00002)\end{array}$ & \\
\hline \multirow[t]{5}{*}{0.20} & 0.01 & 0.0125 & 0.0592 & 0.1816 & 0.0392 & 0.1756 \\
\hline & 0.005 & 0.00589256 & 0.0610 & 0.1821 & 0.0408 & 0.1766 \\
\hline & 0.0025 & 0.003125 & 0.0616 & 0.1824 & 0.0413 & 0.1772 \\
\hline & 0.00125 & 0.00147314 & 0.0622 & 0.1825 & 0.0417 & 0.1775 \\
\hline & 0.001 & 0.000790569 & 0.0622 & 0.1825 & 0.0418 & 0.1775 \\
\hline $\mathrm{MC}$ & & & $\begin{array}{c}0.0624 \\
(0.00009)\end{array}$ & & $\begin{array}{c}0.0418 \\
(0.00007)\end{array}$ & \\
\hline
\end{tabular}

Parameters: $S=1.0, X=0.9, r=0.06, \sigma=0.25, T=1.0$ year.

The grid size for the average price is given by $\Delta Y=\sigma \sqrt{\Delta t} / K$, where $K$ is an integer. The five $\Delta Y \mathrm{~s}$ in the table correspond to $K=2,3,4,6$ and 10 .

MC, values computed using Monte Carlo simulations with $10^{6}$ paths (500,000 plus 500,000 antithetics) and 2,000 time steps in each simulation. The values in parentheses below option prices are standard errors of the MC simulations. 
Table 2: Values of European-style MABOs with monitoring frequency, $F=1-$ comparison of results computed using Monte Carlo simulation, our FSGM and Heritage's (2002) formula

\begin{tabular}{ccccccc}
\hline$D$ & $b$ & MC & FSGM & RelE & Heritage & RelE \\
\hline \multirow{2}{*}{0.2} & 0.10 & 0.0242 & 0.0242 & $-0.04 \%$ & 0.0175 & $-27.69 \%$ \\
& 0.12 & 0.0308 & 0.0306 & $-0.52 \%$ & 0.0240 & $-21.57 \%$ \\
& 0.14 & 0.0378 & 0.0380 & $0.42 \%$ & 0.0315 & $-17.11 \%$ \\
& 0.16 & 0.0456 & 0.0456 & $0.09 \%$ & 0.0399 & $-12.50 \%$ \\
& 0.18 & 0.0539 & 0.0537 & $-0.30 \%$ & 0.0490 & $-8.75 \%$ \\
& 0.20 & 0.0624 & 0.0622 & $-0.39 \%$ & 0.0587 & $-5.63 \%$ \\
\hline \multirow{3}{*}{0.04} & & & & & & \\
& 0.10 & 0.0119 & 0.0119 & $0.25 \%$ & 0.0109 & $-8.40 \%$ \\
& 0.12 & 0.0163 & 0.0163 & $-0.00 \%$ & 0.0154 & $-5.52 \%$ \\
& 0.14 & 0.0216 & 0.0217 & $0.65 \%$ & 0.0208 & $-4.15 \%$ \\
& 0.16 & 0.0277 & 0.0277 & $0.18 \%$ & 0.0270 & $-2.53 \%$ \\
& 0.18 & 0.0344 & 0.0344 & $-0.00 \%$ & 0.0340 & $-1.16 \%$ \\
& 0.20 & 0.0418 & 0.0418 & $-0.05 \%$ & 0.0417 & $-0.24 \%$ \\
\hline \multirow{2}{*}{0.01} & 0.10 & 0.0083 & 0.0083 & $0.67 \%$ & 0.0082 & $-1.96 \%$ \\
& 0.12 & 0.0119 & 0.0120 & $0.49 \%$ & 0.0119 & $-0.81 \%$ \\
& 0.14 & 0.0164 & 0.0166 & $1.11 \%$ & 0.0164 & $-0.92 \%$ \\
& 0.16 & 0.0217 & 0.0218 & $0.72 \%$ & 0.0218 & $0.22 \%$ \\
& 0.18 & 0.0277 & 0.0278 & $0.29 \%$ & 0.0279 & $0.42 \%$ \\
& 0.20 & 0.0345 & 0.0345 & $0.04 \%$ & 0.0348 & $0.85 \%$ \\
\hline
\end{tabular}

Parameters: $S=1.0, X=0.9, r=0.06, \sigma=0.25, T=1.0$ year.

$\mathrm{MC}$, values computed using Monte Carlo simulations with $10^{6}$ paths (500,000 plus 500,000 antithetics) and 2,000 time steps in each simulation. FSGM, values computed using our FSGM with grid sizes $\Delta t=0.001$ and $\Delta Y=0.000790569$. Heritage, values of equation (6) in Heritage (2002). RelE, relative error of FSGM or Heritage computed by using MC results as benchmarks. 
Table 3: Values of American-style MABOs with monitoring frequency, $F=1$ - comparison of results computed using our FSGM and Longstaff and Schwartz's (2001) least-squares Monte Carlo approach

\begin{tabular}{cccccccc}
\hline & \multicolumn{3}{c}{$D=0.2$} & & \multicolumn{3}{c}{$D=0.04$} \\
\cline { 3 - 4 } \cline { 5 - 7 }$b$ & FSGM & LSM & RelE & & FSGM & LSM & RelE \\
\hline \multirow{2}{*}{0.10} & 0.1739 & 0.1625 & $-6.58 \%$ & & 0.1624 & 0.1444 & $-11.10 \%$ \\
& & $(0.0003)$ & & & $(0.0002)$ & \\
0.12 & 0.1765 & 0.1659 & $-6.00 \%$ & & 0.1666 & 0.1492 & $-10.46 \%$ \\
& & $(0.0003)$ & & & $(0.0002)$ & \\
0.14 & 0.1786 & 0.1687 & $-5.52 \%$ & & 0.1703 & 0.1535 & $-9.84 \%$ \\
& & $(0.0003)$ & & & $(0.0002)$ & \\
0.16 & 0.1801 & 0.1708 & $-5.19 \%$ & & 0.1732 & 0.1574 & $-9.12 \%$ \\
& & $(0.0003)$ & & & $(0.0003)$ & \\
0.18 & 0.1814 & 0.1726 & $-4.87 \%$ & 0.1755 & 0.1609 & $-8.34 \%$ \\
& & $(0.0003)$ & & & $(0.0003)$ & \\
0.20 & 0.1825 & 0.1741 & $-4.61 \%$ & 0.1775 & 0.1640 & $-7.62 \%$ \\
& & $(0.0003)$ & & & $(0.0003)$ & \\
\hline
\end{tabular}

Parameters: $S=1.0, X=0.9, r=0.06, \sigma=0.25, T=1.0$ year.

FSGM, converged values (accuracy up to 0.0001) computed using our FSGM with grid sizes $\Delta t=0.001$ and $\Delta Y=0.000790569$. LSM, values computed using Longstaff and Schwartz's (2001) least-squares Monte Carlo approach with 100,000 paths (50,000 plus 50,000 antithetics) and 200 time steps in each simulation. Values in parentheses below option prices are standard errors of the LSM simulations. RelE, relative error of LSM computed by using our converged FSGM results as benchmarks. 
Table 4: Values of continuously monitored European-style MABOs, i.e., $F=\infty$, by extrapolation from the discretely monitored MABO prices computed using our FSGM, and Heritage's (2002) formula (the unit of the prices is $10^{-2}$ )

\begin{tabular}{ccccccccc}
\hline$D$ & $b$ & $V_{\infty}$ & $V_{1}$ & $V_{2}$ & $V_{R E 2}$ & RelE & $V_{H}$ & RelE \\
\hline & \multicolumn{7}{c}{ Case I } \\
\cline { 2 - 10 } 0.2 & 0.10 & 2.202 & 2.442 & 2.287 & 2.236 & $1.53 \%$ & 1.431 & $-35.03 \%$ \\
& 0.12 & 2.825 & 3.082 & 2.908 & 2.850 & $0.89 \%$ & 1.986 & $-29.70 \%$ \\
& 0.14 & 3.518 & 3.788 & 3.600 & 3.537 & $0.54 \%$ & 2.637 & $-25.05 \%$ \\
& 0.16 & 4.277 & 4.553 & 4.355 & 4.289 & $0.28 \%$ & 3.374 & $-21.11 \%$ \\
& 0.18 & 5.095 & 5.365 & 5.162 & 5.094 & $-0.03 \%$ & 4.187 & $-17.83 \%$ \\
& 0.20 & 5.948 & 6.216 & 6.010 & 5.941 & $-0.11 \%$ & 5.061 & $-14.92 \%$ \\
\hline 0.04 & 0.10 & 1.095 & 1.196 & 1.128 & 1.106 & $0.99 \%$ & 0.946 & $-13.74 \%$ \\
& 0.12 & 1.521 & 1.639 & 1.557 & 1.530 & $0.56 \%$ & 1.352 & $-11.08 \%$ \\
& 0.14 & 2.027 & 2.159 & 2.063 & 2.031 & $0.22 \%$ & 1.846 & $-8.91 \%$ \\
& 0.16 & 2.611 & 2.759 & 2.651 & 2.614 & $0.13 \%$ & 2.425 & $-7.14 \%$ \\
& 0.18 & 3.277 & 3.427 & 3.307 & 3.267 & $-0.31 \%$ & 3.081 & $-5.97 \%$ \\
& 0.20 & 4.010 & 4.161 & 4.031 & 3.987 & $-0.56 \%$ & 3.809 & $-5.01 \%$ \\
\hline
\end{tabular}

Case II

\begin{tabular}{ccccccccc}
\cline { 2 - 8 } 0.1 & 0.10 & 0.342 & 0.369 & 0.350 & 0.343 & $0.53 \%$ & 0.084 & $-75.47 \%$ \\
& 0.12 & 0.528 & 0.556 & 0.529 & 0.520 & $-1.52 \%$ & 0.199 & $-62.38 \%$ \\
& 0.14 & 0.769 & 0.835 & 0.799 & 0.787 & $2.37 \%$ & 0.379 & $-50.76 \%$ \\
& 0.16 & 1.062 & 1.125 & 1.081 & 1.067 & $0.41 \%$ & 0.628 & $-40.84 \%$ \\
& 0.18 & 1.401 & 1.456 & 1.405 & 1.388 & $-0.95 \%$ & 0.945 & $-32.54 \%$ \\
& 0.20 & 1.779 & 1.883 & 1.826 & 1.807 & $1.54 \%$ & 1.321 & $-25.74 \%$ \\
\hline 0.05 & 0.10 & 0.178 & 0.193 & 0.182 & 0.178 & $0.44 \%$ & 0.064 & $-63.87 \%$ \\
& 0.12 & 0.307 & 0.324 & 0.307 & 0.301 & $-1.99 \%$ & 0.156 & $-49.29 \%$ \\
& 0.14 & 0.488 & 0.537 & 0.511 & 0.502 & $2.83 \%$ & 0.303 & $-37.97 \%$ \\
& 0.16 & 0.723 & 0.773 & 0.738 & 0.727 & $0.48 \%$ & 0.512 & $-29.26 \%$ \\
& 0.18 & 1.011 & 1.055 & 1.013 & 0.998 & $-1.21 \%$ & 0.782 & $-22.61 \%$ \\
& 0.20 & 1.344 & 1.437 & 1.386 & 1.369 & $1.88 \%$ & 1.111 & $-17.34 \%$ \\
\hline
\end{tabular}

Case I: $S=1.0, X=0.9, r=0.06, \sigma=0.25, T=1.0$ year; $\Delta t=0.001, \Delta Y=0.00395(K=2)$. Case II: $S=1.0, X=1.05, r=0.01, \sigma=0.30, T=0.5$ year; $\Delta t=0.001, \Delta Y=0.00237(K=4)$. $V_{\infty}$, values of the continuously monitored European-style MABOs computed using Monte Carlo simulations with $10^{6}$ paths (500,000 plus 500,000 antithetics) and 2,000 time steps in each simulation. $V_{i}, i=1$ and 2, values of the discretely monitored European MABOs with monitoring frequency, $F=i$, computed using our FSGM. $V_{R E 2}$, values of Richardson's (1910) two-point extrapolation, i.e., $V_{R E 2}=\left(4 V_{2}-V_{1}\right) / 3 . V_{H}$, values of Heritage's (2002) formula (on page 59 in his paper). RelE, relative error of the results from the extrapolation or Heritage computed by using $V_{\infty}$ as benchmarks. 
Table 5: Values of continuously monitored American-style MABOs, i.e., $F=\infty$, by extrapolation from the discretely monitored American MABO prices computed using our FSGM

\begin{tabular}{|c|c|c|c|c|c|c|}
\hline \multirow[b]{3}{*}{$b$} & \multicolumn{6}{|c|}{ Case I } \\
\hline & \multicolumn{3}{|c|}{$D=0.2$} & \multicolumn{3}{|c|}{$D=0.04$} \\
\hline & $V_{1}$ & $V_{2}$ & $V_{R E 2}$ & $V_{1}$ & $V_{2}$ & $V_{R E 2}$ \\
\hline 0.10 & 0.1738 & 0.1716 & 0.1709 & 0.1622 & 0.1610 & 0.1606 \\
\hline 0.12 & 0.1764 & 0.1744 & 0.1738 & 0.1665 & 0.1654 & 0.1651 \\
\hline 0.14 & 0.1784 & 0.1767 & 0.1761 & 0.1700 & 0.1691 & 0.1688 \\
\hline 0.16 & 0.1800 & 0.1786 & 0.1781 & 0.1729 & 0.1722 & 0.1720 \\
\hline 0.18 & 0.1813 & 0.1801 & 0.1797 & 0.1753 & 0.1747 & 0.1745 \\
\hline 0.20 & 0.1824 & 0.1814 & 0.1811 & 0.1773 & 0.1768 & 0.1766 \\
\hline
\end{tabular}

Case II

\begin{tabular}{cccccccc} 
& \multicolumn{3}{c}{$D=0.1$} & & \multicolumn{3}{c}{$D=0.05$} \\
\cline { 2 - 5 } \cline { 6 - 8 }$b$ & $V_{1}$ & $V_{2}$ & $V_{R E 2}$ & & $V_{1}$ & $V_{2}$ & $V_{R E 2}$ \\
\hline & & & & & & & \\
0.10 & 0.0576 & 0.0558 & 0.0553 & & 0.0525 & 0.0510 & 0.0505 \\
0.12 & 0.0602 & 0.0589 & 0.0585 & & 0.0565 & 0.0554 & 0.0550 \\
0.14 & 0.0623 & 0.0615 & 0.0612 & & 0.0598 & 0.0590 & 0.0588 \\
0.16 & 0.0635 & 0.0630 & 0.0628 & & 0.0618 & 0.0612 & 0.0611 \\
0.18 & 0.0643 & 0.0640 & 0.0639 & & 0.0632 & 0.0628 & 0.0627 \\
0.20 & 0.0649 & 0.0647 & 0.0647 & & 0.0642 & 0.0640 & 0.0639 \\
\hline
\end{tabular}

Case I: $S=1.0, X=0.9, r=0.06, \sigma=0.25, T=1.0$ year; $\Delta t=0.001, \Delta Y=0.00395(K=2)$. Case II: $S=1.0, X=1.05, r=0.01, \sigma=0.30, T=0.5$ year; $\Delta t=0.001, \Delta Y=0.00237$ $(K=4)$.

$V_{i}, i=1$ and 2 , values of the discretely monitored American-style MABOs with monitoring frequency, $F=i$, computed using our FSGM. $V_{R E 2}$, values of the Richardson's (1910) two-point extrapolation, i.e., $V_{R E 2}=\left(4 V_{2}-V_{1}\right) / 3$. 
Table 6: Values of European-style and American-style MABOs when the spot price, $S$, is close to the barrier level, $H$, i.e., when $b=\ln (H / S)$ is small

\begin{tabular}{|c|c|c|c|c|c|c|}
\hline & \multicolumn{6}{|c|}{ European MABO $\left(\times 10^{-3}\right)$} \\
\hline & \multicolumn{3}{|c|}{$D=0.2$} & \multicolumn{3}{|c|}{$D=0.04$} \\
\hline & $V_{1}$ & $V_{2}$ & $V_{\infty}$ & $V_{1}$ & $V_{2}$ & $V_{\infty}$ \\
\hline FSGM & 4.8992 & 4.5198 & 4.3933 & 1.1695 & 1.0470 & 1.0062 \\
\hline \multirow[t]{2}{*}{$\mathrm{MC}$} & 4.9371 & 4.5981 & 4.3901 & 1.1902 & 1.0789 & 1.0170 \\
\hline & \multicolumn{6}{|c|}{ American MABO } \\
\hline FSGM & 0.1512 & 0.1493 & 0.1487 & 0.1285 & 0.1270 & 0.1265 \\
\hline
\end{tabular}

Parameters: $S=1.0, b=0.01, X=0.9, r=0.06, \sigma=0.25, T=1.0$.

$V_{i}, i=1,2$ and $\infty$, values of the MABOs with monitoring frequency, $F=1,2$ and $\infty$. FSGM, values computed using our FSGM with $\Delta t=0.001$ and $\Delta Y=0.00395$ for $F=1$ and 2 , and using the Richardson's (1910) two-point extrapolation, $V_{R E 2}=\left(4 V_{2}-V_{1}\right) / 3$, for $F=\infty$. MC, values computed using Monte Carlo simulations with $10^{6}$ paths (500,000 plus 500,000 antithetics) and 2, 000 time steps in each simulation. 